\title{
Experimental program of the Super-FRS Collaboration at FAIR and developments of related instrumentation
}

\author{
Äystö, J.
}

2016-06-01

Äystö , J , Behr , K -H , Benlliure , J , Bracco , A, Egelhof , P, Fomichev , A, Gales , S , Geissel , H , Grahn , T , Grigorenko , L V , Harakeh, M N , Hayano , R , Heinz , S , Itahashi , $\mathrm{K}$, Jokinen , A , Kalantar-Nayestanaki , N , Kanungo , R, Lenske , H , Mukha , I, Muenzenberg, G, Nociforo , C , Ong , H J , Pietri , S , Pfutzner , M , Plass , W , Prochazka , A, Purushothaman , S , Saito , T , Scheidenberger , C , Simon , H, Tanihata , I, Terashima , S , Toki , H , Trache , L, Weick, H, Winfield, J S, Winkler, M , Zamfir , V \& Super-FRS Collaboration NUSTAR 2016, ' Experimental program of the Super-FRS Collaboration at FAIR and developments of related instrumentation ', Nuclear Instruments \& Methods in Physics Research. Section B: Beam Interactions with Materials and Atoms, vol. 376 , pp. 111-115 . https://doi.org/10.1016/j.nimb.2016.02.042

http://hdl.handle.net/10138/232948

https://doi.org/10.1016/j.nimb.2016.02.042

cc_by_nc

acceptedVersion

Downloaded from Helda, University of Helsinki institutional repository.

This is an electronic reprint of the original article.

This reprint may differ from the original in pagination and typographic detail.

Please cite the original version. 


\title{
Experimental program of the Super-FRS Collaboration at FAIR and developments of related instrumentation
}

\author{
J. Äystö ${ }^{\mathrm{a}}$, K.-H. Behr ${ }^{\mathrm{b}}$, J. Benlliure ${ }^{\mathrm{c}}$, A. Bracco ${ }^{\text {d }}$, P. Egelhof ${ }^{\mathrm{b}}$, A. Fomichev $^{\mathrm{e}}$, S. Galès $^{\mathrm{f}, \mathrm{p}}$, H. Geissel $^{\mathrm{b}, \mathrm{l}}$, T. Grahn $^{\mathrm{a}, \mathrm{j}}$, \\ L.V. Grigorenko ${ }^{\mathrm{e}}$, M.N. Harakeh ${ }^{\mathrm{g}}$, R. Hayano ${ }^{\mathrm{h}}$, S. Heinz ${ }^{\mathrm{b}}$, K. Itahashi ${ }^{\mathrm{i}}$, A. Jokinen ${ }^{\mathrm{a}, \mathrm{j}}$, N. Kalantar-Nayestanaki ${ }^{\mathrm{g}}$, R. \\ Kanungo $^{\mathrm{k}}$, H. Lenske ${ }^{\mathrm{l}}$, I. Mukha ${ }^{\mathrm{b}, *}$, G. Münzenberg ${ }^{\mathrm{b}}$, C. Nociforo ${ }^{\mathrm{b}}$, H.J. Ong ${ }^{\mathrm{m}}$, S. Pietri $^{\mathrm{b}}$, M. Pfützner ${ }^{\mathrm{n}}$, W. Plaß ${ }^{\mathrm{b}}$,

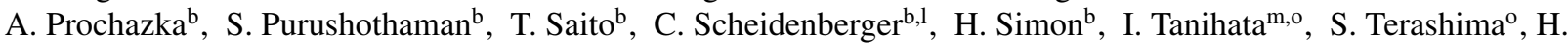 \\ Toki $^{\mathrm{m}}$, L. Trache ${ }^{\mathrm{p}}$, H. Weick ${ }^{\mathrm{b}}$, J.S. Winfield ${ }^{\mathrm{b}}$, M. Winkler ${ }^{\mathrm{b}}$, V. Zamfir ${ }^{\mathrm{p}}$, for the Super-FRS Collaboration of \\ NUSTAR@FAIR

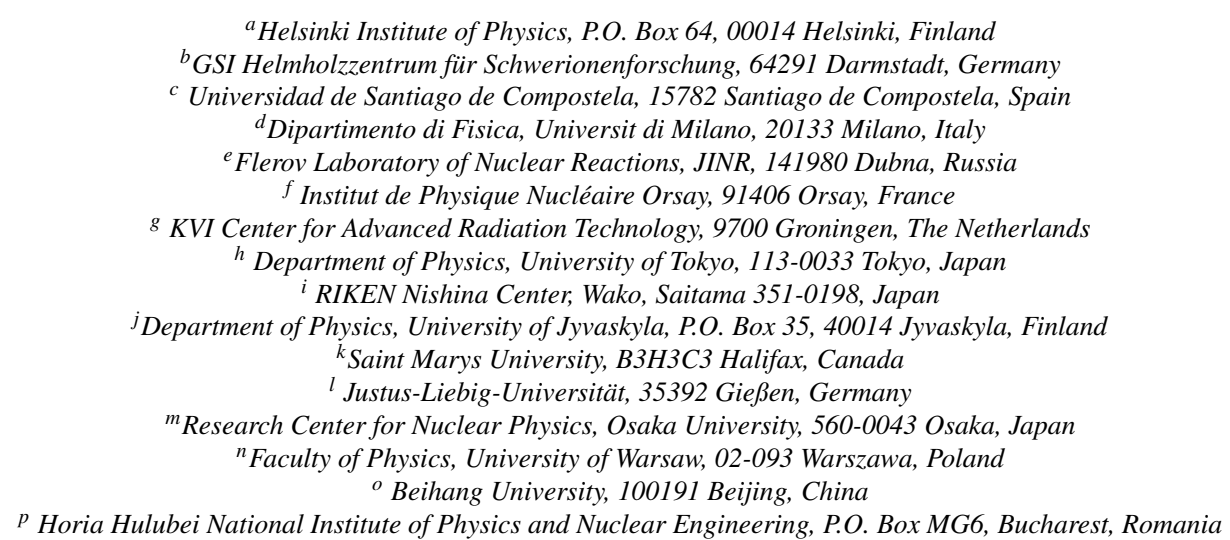

\begin{abstract}
The physics program at the super-conducting fragment separator (Super-FRS) at FAIR, being operated in a multiplestage, high-resolution spectrometer mode, is discussed. The Super-FRS will produce, separate and transport radioactive beams at high energies up to $1.5 \mathrm{AGeV}$, and it can be also used as a stand-alone experimental device together with ancillary detectors. Various combinations of the magnetic sections of the Super-FRS can be operated in dispersive, achromatic or dispersion-matched spectrometer ion-optical modes, which allow measurements of momentum distributions of secondary-reaction products with high resolution and precision. A number of unique experiments in atomic, nuclear and hadron physics are suggested with the Super-FRS as a stand-alone device, in particular searches for new isotopes, studies of hypernuclei, delta-resonances in exotic nuclei and spectroscopy of atoms characterized by bound mesons. Rare decay modes like multiple-proton or neutron emission and the nuclear tensor force observed in high-momentum regime can be also addressed. The in-flight radioactivity measurements as well as fusion, transfer and deep-inelastic reaction mechanisms with the slowed-down and energy-bunched fragment beams are proposed for the high-resolution and energy buncher modes at the Super-FRS.
\end{abstract}

Keywords: Relativistic energy, projectile fragments, exotic nuclei, high-resolution magnetic spectrometer, Super-FRS, momentum measurement, isotopes, atomic collisions, mesonic atoms, hyper-nuclei, tensor force, delta resonances, nuclear radii, in-flight decay, proton radioactivity, neutron radioactivity, active target, reactions PACS: codes 29.20.db, 21.10.Dr, 29.30.Aj

\section{Introduction}

${ }^{*}$ Corresponding author. E-mail address: I.Mukha@gsi.de Preprint submitted to Nuclear Instruments and Methods B
The Super-FRS is one of the main scientific instruments of the future FAIR facility in Darmstadt, Ger- 


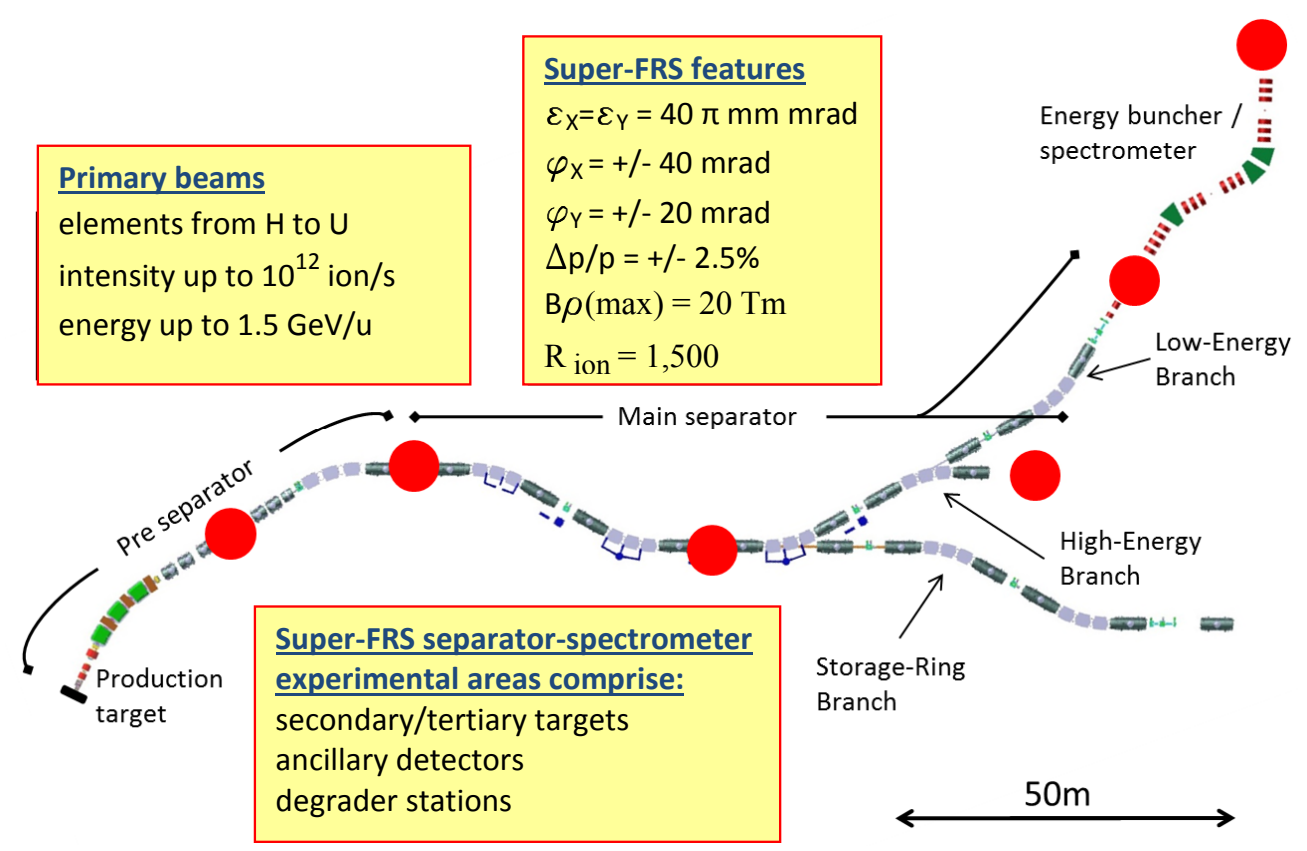

Figure 1: Scheme of the Super-FRS with its three branches and the low-energy-buncher system. The experimental areas of the Super-FRS collaboration [7] are indicated by the red-filled circles.

many. This magnetic high-resolution spectrometer, coupled to the heavy-ion synchrotron complex SIS-18/ SIS100 , will be the central device of the NUSTAR collaboration for research with exotic nuclei $[1,2]$. The separator will provide beams of exotic nuclei ranging from hydrogen up to uranium over a broad energy range equivalent to a maximum magnetic rigidity of up to $20 \mathrm{Tm}$. The exotic nuclei are produced via projectile fragmentation, fission and two-step reactions. The nuclei of interest will be separated in flight and delivered to the large-scale detector systems which will be placed beyond the three separator branches: HISPEC/DESPEC and MATS/LaSPEC at the Low-Energy Branch (LEB) [3], $\mathrm{R}^{3} \mathrm{~B}$ at the High-Energy Branch [4] and ILIMA at the Storage-Ring Branch [5], see Fig. 1.

The Super-FRS can be also used as a stand-alone experimental device. In this function, the Super-FRS is a powerful and versatile high-resolution spectrometer system for exotic nuclei over a large energy range up to 1.5 AGeV, which makes it a worldwide unique instrument, see Fig. 2. The various magnetic sections of the Super-FRS can be operated as dispersive, achromatic or dispersion-matched spectrometer units which are wellsuited for the measurements of momentum distribution of reaction products with high resolution and precision. With this flexibility, the Super-FRS allows for a variety of novel experiments at relativistic energy. Taking advantage of new stages and ion-optical modes, it also allows for an extension of preceding experiments at the existing fragment separator FRS [6].

The physics program of the Super-FRS Experiment Collaboration can be found in Ref. [7]. It was also highlighted in Refs. [8, 9]. The planned experiments are the search for new isotopes and measurements of their production cross sections, inclusive reaction and nuclear radii measurements, basic atomic collision studies, searches for hypernuclei, $\Delta$-resonances in exotic nuclei and the spectroscopy of atoms with bound mesons. Exotic decays like multiple-nucleon emission and the influence of the nuclear tensor force in the observation of high-momentum components of the nucleons are also addressed. The in-flight decay measurements of unbound nuclei in the picosecond time range, pioneered at the FRS, will be extended. The studies of fusion, transfer and deep-inelastic reactions with the slowed-down and energy-compressed beams are proposed for the high-resolution energy-buncher at the LEB of SuperFRS [3]. All research topics are described in Ref. [7] where all members of the collaboration are listed.

\section{Experiments and related detection techniques}

All suggested experiments will use standard beam detectors of the Super-FRS: position-tracking time- 


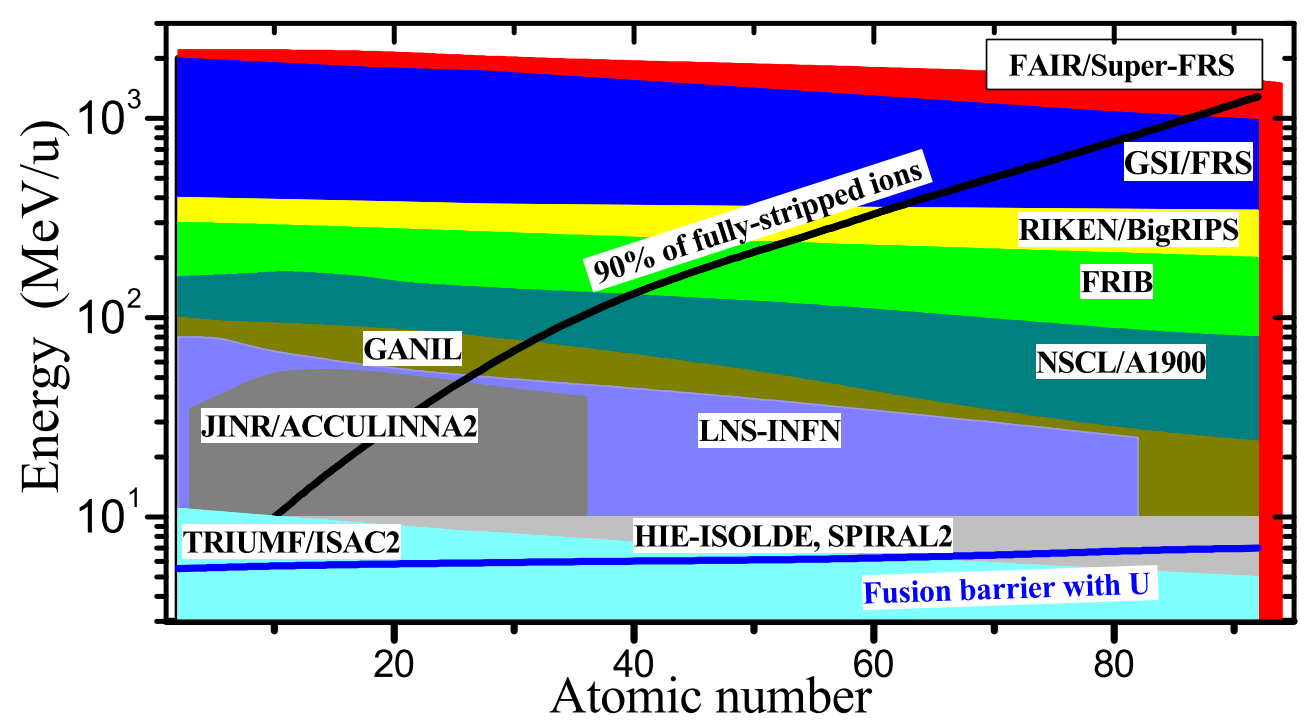

Figure 2: Energy and element ranges covered by existing and planned radioactive beam facilities (the filled histograms labeled by the facility abbreviations). The black line shows energies at which $90 \%$ of accelerated ions can be fully-stripped of electrons. The blue line shows fusion barriers of accelerated ions with uranium target.

projection chambers with GEM technology [10, 11], time-of-flight (ToF) detectors [12, 13, 14, 15], energyloss detectors [16, 17], whose developments are based on the existing FRS detectors.

\subsection{Search for new isotopes}

The search for the limits of existence of nuclei is one of the most essential studies in nuclear physics. It is strongly connected with the search for new isotopes. Identification of new isotopes and measurements of their production cross sections are essential for planning of all NUSTAR experiments. Regions of main interest are located between $\mathrm{Sn}$ and U, where even the heaviest fragments are fully stripped because of high energy and therefore can be identified unambiguously, even when very rarely produced.

\subsection{Atomic collision studies}

The accurate knowledge of the atomic interaction of heavy ions penetrating through matter is essential for the successful operation of the Super-FRS. Also, the complete slowing down in a gas-filled stopping cell at the LEB of Super-FRS is a challenging task because of the large range distribution of the fragments compared to the typical thickness of a helium-filled stopping cell $[18,19]$. In the Super-FRS energy domain, data for stopping powers, energy and angular straggling and charge-state distributions of heavy ions are scarce. Therefore, basic atomic collision processes have to be measured in an early stage of the Super-FRS operation. The high momentum-resolution and charge-state separation capabilities of the Super-FRS suggest the solid basis for such studies. Some special effects, characteristic for relativistic heavy ions, are on the agenda of this collaboration too: the emission of Vavilov-Cherenkov radiation [20] and the Resonant Coherent Excitation (RCE) in crystals (nuclear RCE, or the Okorokov effect). The Okorokov effect was predicted in 1966 [21] and has not been observed yet. Either highly relativistic ion beams $(\gamma \gg 1)$ are needed to reach high excitation energies $(\sim \mathrm{MeV})$ or low-lying long-lived states $(\sim 10 \mathrm{keV})$ are required in the nuclei to be excited. Radioactive ions exhibit a widespread range of excitation energies, transitions and lifetimes [22]. Depending on thickness of the crystal and other experimental parameters one can obtain excitation probabilities which are orders of magnitude higher than in amorphous material. The feasibility of channeling experiments has been already demonstrated with pilot experiments at the FRS [23]. The critical angle, which is necessary for channeling conditions, can be achieved with a special ionoptical setting of the Super-FRS.

\subsection{Strangeness in exotic nuclei}

Strangeness in nuclei opens up a new degree of freedom to study the strong force. The production of hypernuclei by coalescence, where the $\Lambda$ is produced in peripheral collisions and sticks to the projectile-like fragment, has been already demonstrated at the GSI facility 
by the HypHI collaboration at energies of $1-2 \mathrm{AGeV}$ [24]. Since this mechanism is universal and works also with exotic nuclei, a drastic expansion of the hypernuclear chart is expected and the third dimension of the chart of nuclei is opened up. The invariant mass of the final state, for example a $\pi^{-}$and a nuclear fragment after weak hypernuclei decay, gives an effective signature for identifying a hypernucleus. With the momentum resolution of the Super-FRS, one can expect a mass resolution of $<1 \mathrm{MeV} / \mathrm{c}^{2}$. Also, the half-life of a hypernucleus can be determined from the flight path distribution behind the production target when decays. Heavy-ion collisions also provide a possibility for forming multi- $\Lambda$ hypernuclei.

\subsection{Delta-resonances for probing nuclear structure}

In high-energy heavy-ion collisions, in particular in charge-exchange reactions, there is clear evidence for $\Delta$-resonance in nuclei. The $\Delta$-resonance is a $\Delta S=1$, $\Delta \mathrm{I}=1$ spin- and isospin-flip intrinsic excitation of the nucleon. It is a partner of the corresponding $\Delta S=1, \Delta \mathrm{I}=1$ excitation of the nucleus, known as the Gamow-Teller resonance. The Super-FRS presents unique possibilities to study the $\Delta$ and other-baryon resonances both in stable and in exotic nuclei. Pilot experiments of isobaric charge-exchange reactions have already been performed with the FRS [25]. Heavy-ion collisions also provide opportunities to study two $\Delta \mathrm{s}$ in nuclei as well as $\Delta-\Delta$ interactions. The proposed experiments on $\Delta$ excitations in exotic nuclei can be realized by using the Super-FRS with its standard beam detectors measuring the momentum distribution of the isobaric nuclides created via charge-exchange reactions. One could also apply a setup providing $4 \pi$-detection capability and tracking of all charged pions in the magnetic field, e.g., the WASA detector [26].

\subsection{The tensor forces in nuclei}

Recent ab-initio calculations of light nuclei demonstrate that about $80 \%$ of nuclear binding is due to pions, and the tensor force is as important as the central forces in the pion-exchange interactions [27, 28]. However, the tensor force has not been explicitly handled in nuclear models except for the lightest nuclei. Moreover, recent studies of nuclei far from the stability valley show the importance of the tensor forces through changes of magic numbers and, e.g., a peculiar mixing of $s$ - and $p$ waves in the neutron-halo nucleus ${ }^{11} \mathrm{Li}$. An important property of the tensor force is that it produces a strong proton-neutron attraction ( $p-n$ pairing) in a nucleus and introduces high-momentum nucleons in nuclei. Most of nuclear models are based on the mean-field approach and do not include the $p-n$ pairing correlations. Thus, the observation of high-momentum components of nucleons and correlated nucleons in nuclei are essential to clarify the importance of the tensor force. Zero-degree scattering is the best way to get rid of possible difficulties from reaction mechanisms. The Super-FRS is the only facility in the world that provides high-energy incident beams exceeding $400 \mathrm{AMeV}$ with a high-resolution zero-degree spectrometer mode. Nucleon-transfer reactions such as $(\mathrm{p}, \mathrm{d}),(\mathrm{d}, \mathrm{t})$, and $\left(\mathrm{d},{ }^{3} \mathrm{He}\right)$ are relevant for this purpose. In a pilot experiment at the FRS in 2014, $(p, d)$ reactions have been studied and preliminary results demonstrated sensitivity to the tensor forces.

\subsection{Pionic atoms}

The discovery of deeply-bound pionic states in heavy atoms at FRS has opened up a new field of fundamental studies of the meson-nucleus interactions, which contribute to the understanding of the non-trivial structure of the vacuum of quantum chromodynamics (QCD) $[29,30]$. The experiments on the meson-nucleus bound system will concentrate on the existence of the states and on possible modification of meson properties inside nuclear matter. The results will help to answer the key question of partial restoration of chiral symmetry breaking, which is related to the unknown process of mass evolution. The experiments employ transfer reactions with protons and deuterons and then look for bound states in missing-mass spectra. The high-momentum resolving power and the independent multiple-stage operation of the Super-FRS ion-optical system are essential key features for these experiments. The $(\mathrm{p}, \mathrm{d})$ reaction at $2.5 \mathrm{GeV}$ is suitable for producing $\eta^{\prime}$-bound nuclei. A first pilot experiment on $\eta^{\prime}$ bound nuclei has been carried out in 2014 [31].

\subsection{Nuclear radii and momentum distributions}

The proposed experiments combine measurements of nuclear-interaction and charge-changing cross sections with nucleon-removal and high-resolution momentum distribution measurements. Such studies are aimed for new structures in nuclei, in particular in heavier mass regions $(A>50)$. Also the thickness of neutron skins is of interest, and this method is one of the sensitive ways to determine the asymmetry term of the equation of state (EOS) of asymmetric nuclear matter. The EOS for asymmetric nuclear matter is of utmost importance for understanding the stellar objects (such as neutron stars) and their dynamic changes (such as supernovae). The method can be applied for very short-lived nuclei 


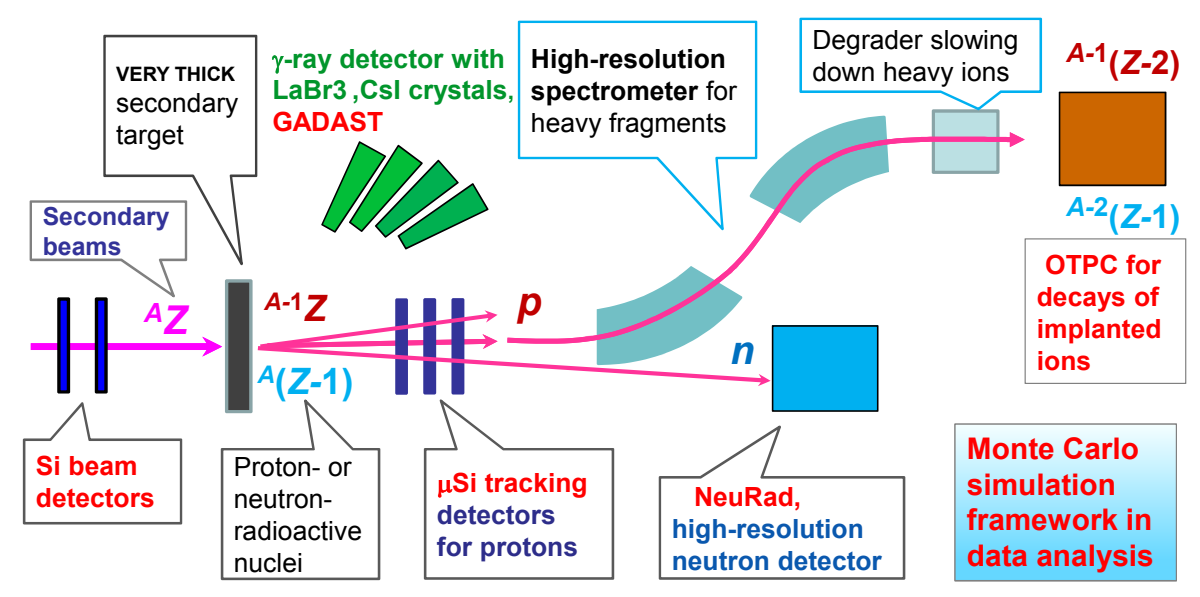

Figure 3: Schematic view of detectors which allow to study exotic radioactive decays with a secondary target located at the middle focal plane of the Super-FRS main separator. High-resolution angular correlations of decay products are planned for proton and neutron radioactive precursors populated by secondary beams, see Ref. [39].

and weak-intensity beams and thus has the possibility to reach the most neutron-rich isotopes [32].

\subsection{Low-momentum transfer experiments with an ac- tive target}

Unique information on matter distribution and radii of exotic nuclei can be obtained by proton or alpha scattering with low-q transfer using active targets. In such a measurement, a low-energy recoil particle can be detected in coincidence with the forward-emitted residual nucleus to identify the reaction channel. An application of an active target at the Super-FRS (like the IKAR active target [33] which was successfully used for experiments on light halo nuclei $[34,35])$ may profit from the fact that the scattered beam-like particle may be detected with a momentum resolution down to $10^{-4}$ in the Super-FRS section downstream of the active target.

\subsection{Radioactive decays in-flight}

The nuclear landscape is characterized by two borders between nuclides with bound and un-bound nucleons (the proton and neutron drip-lines), and one of the major goals of modern nuclear physics is establishing a map of nucleon-stable nuclei. However, nuclear systems beyond the drip-lines demonstrate individual states too. For example, the nuclei with small decay energies are radioactive with half-lives longer than $10^{-15}$ $10^{-12} \mathrm{~s}$. Such nuclear decays via emission of proton(s) or neutron(s) are called proton radioactivity and neutron radioactivity, respectively. The unbound nuclei with shorter half-lives called resonances still have individual nuclear structure. The unbound nuclei can be studied in nuclear reactions either by invariant-mass method or, alternatively, by their decays in-flight by tracking trajectories of decay fragments and reconstructing their decay vertices. Outside the proton drip-line, proton radioactivity prevails and some nuclides with two-proton $(2 p)$ emissions have been observed. They allow studying $2 p$ correlations in nuclei. Four- and six- proton decays are also expected in the extremely proton-rich nuclei. Neutron $(n)$ radioactivity has not been observed yet, mainly due to the fact that the drip line is reached only for light isotopes where only low angular-momentum orbitals are involved, and thus the centrifugal barrier is not high enough to retard the decay. Because of the $n-n$ pairing interaction, some $2 n$-precursors are expected to be stable in respect of $1 n$ emission. Thus their decays should have much longer half-lives due to higher threebody centrifugal barriers [36]. If the $2 n$ radioactivity exists then $n-n$ correlations in nuclei could be studied in a direct way (like Couper pairs). This idea has originated from the previous experiments on $2 p$-radioactivity at GSI where in-flight decays of high-energy radioactive ions had been detected with particle tracking technique $[37,38]$. The Super-FRS experiments intend to use its high-resolution spectrometer capabilities for the outgoing precursor-like decay product and to equip the focal planes with dedicated particle and radiation detectors as displayed in Fig. 3. This setup called EXPERT (EXotic Particle Emission and Radioactivity by Tracking) is described in Ref. [39] in details. Complementary, $2 p$ radioactivity can be studied using an optical TPC placed at the final focus of the Super-FRS [40]. These two detection schemes may utilize the same radioactive beam at the same time [38], and they can cover half-life ranges from $\sim 1 \mathrm{ps}$ to $100 \mathrm{~ns}$ with in-flight decay technique and 
$\sim 100 \mathrm{~ns}$ to $1 \mathrm{~s}$ with implantation-decay method.

\subsection{Nuclear reactions with slowed-down beams}

Reaction studies at Coulomb barrier energies and new isotope production with light neutron-rich radioactive ion beams is one of the topics at the Super-FRS. The in-flight technique will be applied for the production of light exotic nuclei, such as $\mathrm{C}, \mathrm{O}$ or $\mathrm{Ne}$, with ion intensities up to $10^{9} \mathrm{~s}^{-1}$. For these nuclei, the Super-FRS will be superior to ISOL facilities. Furthermore, beams of all elements, independent from their chemical properties, will be available at the Super-FRS. Deep-inelastic transfer, quasi-fission or complete fusion reactions could be applied, e.g., for the production of new n-rich isotopes in the region $\mathrm{Z} \sim 100-105$. These nuclides cannot be produced in fusion reactions with stable beams. Radioactive ion beams provide the possibility for systematic reaction studies in a wide range of projectile isospin, binding energy, deformation and other degrees of freedom. Because of the relatively large cross-sections of up to $100 \mathrm{mb}$, the experiment can be performed in the LEB with secondary beams of intensity $\sim 10^{6} \mathrm{~s}^{-1}$. The Energy Buncher [3] will reduce energy spread of beams on the secondary target. The reaction products will be identified by ToF-E with, e.g., the CORSET detector [41]. Alternatively, one can make use of the rather large energy spread of the slowed-down beams by using a stack of multiple targets, leading to a sliding energy sweep across the excitation functions of all reaction channels. An IGISOL-like system which makes efficient use of the small production rate, is proposed [42]. A following MR-TOF mass spectrometer is an excellent tool for the diagnosis and identification of the ions extracted from the gas cell, for isobar separation, mass measurement and the preparation of ion-bunches to further experimental stations, exploring decay spectroscopy, chemistry etc [43].

\section{Summary}

The Super-FRS at FAIR will be a versatile, multiplestage spectrometer that operates in the beam energy domain above $400 \mathrm{AMeV}$. These features open a wide perspective for a variety of novel and unique experiments in atomic, nuclear and hadron physics with stable and exotic nuclei far beyond the year 2022 .

Acknowledgements. Authors thank O. Kiselev for a helpful contribution during manuscript preparations.

[1] H. Geissel et al., Nucl. Instr. Meth. Phys. Res., B204 (2003) 71.

[2] M. Winkler et al., Nucl. Instr. Meth. Phys. Res., B266 (2008) 4183 .
[3] H. Geissel et al., Nucl. Instr. Meth. Phys. Res., B317 (2013) 277.

[4] H. Scheit, GSI Scientific Report 2012, PHW-ENNA-EXP-57, p.187, http://repository.gsi.de/record/52876.

[5] Yu.A. Litvinov et al., Nucl. Instr. Meth. Phys. Res., B317 (2013) 603.

[6] H. Geissel et al., Nucl. Phys. A701 (2002) 259c.

[7] "Scientific program of the Super-FRS Collaboration: Report of the collaboration to the FAIR management", GSI Report 20144, doi:10.15120/GR-2014-4.

[8] C. Scheidenberger et al., Proc. Int. Nucl. Phys. Conf. (INPC) 2013, v.2 Book Series: EPJ Web of Conf., 66 (2014) 11034.

[9] J. Äystö et al., Proc. Conf. Advances in Radioactive Isotope Science (ARIS2014), JPS Conf. Proc., 6 (2015) 020035.

[10] F. Garcia et al., GSI Scientific Report 2014, p.140, DOI: 10.15120/GR-2015-1-MU-NUSTAR-FRS-04.

[11] F. Garcia et al., in 2012 IEEE Nuclear Science Symposium and Medical Imaging Conference Record (NSS/MIC) N14-121, p. 1119.

[12] O. Kiselev et al., GSI Scientific Report 2012, PHW-ENNAEXP-42, p.172; http://repository.gsi.de/record/52876.

[13] O. Kiselev et al., GSI Scientific Report 2014, p.137, DOI: 10.15120/GR-2015-1-MU-NUSTAR-FRS-03.

[14] F. Schirru et al., GSI Scientific Report 2014, p.502, DOI: 10.15120/GR-2015-1-FG-SFRS-06.

[15] C. Nociforo, Journal of Instrumentation, 9 (2014) C01022.

[16] I. Kaufeld et al., GSI Scientific Report 2013, FG-SFRS-10, p.345; https://repository.gsi.de/record/83425.

[17] S. Maurus et al., GSI Scientific Report 2014, p.143, DOI: 10.15120/GR-2015-1-MU-NUSTAR-FRS-06.

[18] W.R. Plass et al., Nucl. Instr. Meth. Phys. Res., B317 (2013) 457.

[19] M. Ranjan et al., Nucl. Instr. Meth. Phys. Res., A770 (2015) 87.

[20] I.E. Tamm, I.M. Frank, Doklady AN SSSR 14 (1937) 107.

[21] V.V. Okorokov, Sov. Nucl. Phys. 2 (1966) 719.

[22] Yu.L. Pivovarov et al., Nucl. Instr. Meth. B119 (1996) 283

[23] D. Dauvergne et al., Phys. Rev. A59 (1999) 2813.

[24] C. Rappold et al., Nucl. Phys. A 913 (2013) 170.

[25] J. Benlliure et al., private communication.

[26] C. Bargholtz et al., Nucl. Instr. Meth. Phys. Res. A594 (2008) 339.

[27] K. Horii et al., Prog. Theor. Phys. 6 (2012) 127.

[28] H.J. Ong et al., Phys. Lett. B725 (2013) 277.

[29] T. Yamazaki et al., Z. Phys. A355 (1996) 219.

[30] K. Itahashi et al., Prog. Theor. Phys. 128 (2012) 601.

[31] H. Fujioka et al.,Acta Phys. Pol. B 46 (2015) 127.

[32] R. Kanungo, Phys. Scr. T152 (2013) 014002.

[33] G.D. Alkhazov et al., Phys. Rev. Lett. 78 (1997) 2313.

[34] A.V. Dobrovolsky et al., Nucl. Phys. A766 (2006) 1.

[35] S. Ilieva et al., Nucl. Phys. A875 (2012) 8.

[36] L.V. Grigorenko, I. Mukha, C. Scheidenberger, M.V. Zhukov, Phys. Rev. C 84 (2011) 021303.

[37] I. Mukha et al., Phys. Rev. Lett. 99 (2007) 182501.

[38] I. Mukha et al., Phys. Rev. Lett. 115 (2015) 202501.

[39] H. Geissel et al., Proc. Int. Symp. on Exotic Nuclei EXON2014, World Scientific, 2015, ISBN 978-981-4699-45-7, p. 579.

[40] M. Pfutzner et al., Eur. Phys. J. A14 (2002) 279.

[41] E.M. Kozulin et al., Instr. Exp. Tech. 51 (2008) 44.

[42] G. Munzenberg et al., Proc. Int. Symp. EXON2014, World Scientific, 2015, ISBN 978-981-4699-45-7, p. 541.

[43] T. Dickel et al., Nucl. Instr. Meth. Phys. Res. A777 (2015) 172. 